\title{
The Dressing Field Method of Gauge Symmetry Reduction: Presentation and Examples.
}

\author{
Jeremy Attard
}

\begin{abstract}
This paper is a presentation of a recent method of gauge symmetry reduction, distinct from the well-known gauge fixing, the bundle reduction theorem or even the Spontaneous Symmetry Breaking Mechanism (SSBM). Given a symmetry group $G$ acting on a fiber bundle and its naturally associated fields (Ehresmann (or Cartan) connection, curvature, etc.) there are situations where it is possible to erase (in whole or in part) the $G$-action by just reconfiguring these fields, i.e. by making a mere change of field variables in order to get new composite fields on which $G$ (or a subgroup) does not act anymore. Two examples are presented in this paper: the re-interpretation of the BEGHHK (Higgs) mechanism without calling on a SSBM, and the top-down construction of Tractor and Twistor bundles and connections in the framework of conformal Cartan geometry.
\end{abstract}

Mathematics Subject Classification (2010). Primary $53 Z 05$.

Keywords. Gauge theory, Symmetry reduction, Spontaneous symmetry breaking, Higgs mechanism, Twistors.

\section{Introduction}

The fundamental interactions are described in the framework of gauge theories, the geometric content of which is a principal fibre bundle $P$ over a smooth (spacetime) manifold $M$, with structure Lie group $G$, together with associated vector bundles $E=P \times_{\rho} \mathbb{V}$ where $\rho$ is a representation of $G$ on the vector space $\mathbb{V}$. A (classical) matter field is then represented by a section $\xi$ of $E$, while the interacting bosons fields are connections $\omega$ on $P$, and act on matter fields via the associated covariant derivative $D \xi=d \xi+\rho(\omega) \xi$. The curvature associated to a connection $\omega$ will be denoted $\Omega:=d \omega+\frac{1}{2}[\omega, \omega]$. These notations will be used throughout the paper. 
The central notion of a gauge theory is that of local symmetry. The latter is implemented by the local action of $G$, i.e. by the action of the (infinite dimensional) gauge group $\mathfrak{G}=\{g: M \rightarrow G\}$.

Fields $\xi$ and connections $\omega$ transform under the action of $g \in \mathfrak{G}$ as $\omega^{g}=g^{-1} \omega g+g^{-1} d g$ and $\xi^{g}=\rho(g)^{-1} \xi$. Knowing this, one can then write a physical theory by choosing a functional integral $S[\omega, \xi]$ which has the property to be invariant under this action. The theory is said to be gauge invariant. The physical meaning of this feature is that two fields in the same gauge orbit are physically equivalent, i.e. are indistinguishable by any physical experiment. The gauge symmetry is the translation of an intrinsic mathematical redundancy of our formalism.

The description of fundamental interactions on which modern physics is built reduces, then, to the choice of symmetry Lie groups. Electroweak and strong interactions are ruled by the Lie group $U(1) \times S U(2) \times S U(3)$. Regarding the gravitational interaction, the fundamental symmetry group of General Relativity (GR) is the group of diffeomorphisms of the base manifold. Let us remark that one can also write GR under the form of a gauge theory ${ }^{1}$, in which is added a local symmetry ruled by the local action of the Lorentz group $\mathrm{SO}(1,3)$. This can be done in the framework of Cartan geometry.

Although the symmetry group $G$ is central and unavoidable in the construction of a gauge theory, one often needs to reduce its action, i.e. passing to a theory with less symmetry. There can be several reasons for that. For example, for a quantization purpose: the gauge symmetry group produces infinities in the path integral over all fields. Also, e.g. in the case of the electroweak sector of the Standard Model $(\mathrm{SM})(G=U(1) \times S U(2))$, the constraint imposed by the symmetry group is such that mass terms are not allowed, $a$ priori, in the action. Thus, since massive particles are observed, one has to find a way to re-write the same theory but with a smaller symmetry group.

There exists many well-known ways of reducing a symmetry. The simplest is gauge fixing: since all fields in a given gauge orbit are equivalent, one just can choose a particular one - which renders the computations easier, for example; the physical results should be, by definition, independent of the choice of gauge. Another one, which applies in the case of the electroweak sector, as one shall see, is the spontaneous symmetry breaking. In this case, the symmetry reduction is thought as a physical phenomenon, like a phase transition, induced by the fact that the ground state has less symmetry than the theory of which it is a solution.

The recent method of symmetry reduction presented in this paper is called the dressing field method. It is a systematic way of finding, if they exist, new fields which are invariant under the action of the gauge group $\mathfrak{G}$ or one of its subgroup. This method turns out to be a mere change of field variables. This change is performed with the help of a dressing field $u$ which does not, in general, belong to the gauge group $\mathfrak{G}$. Thus, it is neither a gauge transformation nor a gauge fixing: the new field variables, called

\footnotetext{
${ }^{1}$ In the sense of using a connection on a fibre bundle.
} 
composite fields, belong in general to representation spaces - for the action of the remaining gauge (sub)group - different than the original variables.

The aim of the present paper is first to present in a nutshell the formalism of the method and then to give two examples of application. The first one is the reinterpretation of the spontaneous symmetry breaking in the electroweak sector of the SM as being a dressing field symmetry reduction. This gives a new physical interpretation to the BEGHHK (Higgs) mechanism. The second one is the reconstruction of Twistors (and Tractors), in the field of conformal geometry, starting from the conformal Cartan geometry and applying the dressing field to erase a part of the conformal group to end up with the transformations found in the usual constructions. This offers a new insight into the geometric nature of these objects. These are examples among many others. The interested reader will find a more complete and detailed presentation of the dressing field method and its applications in [4].

\section{The dressing field method in a nutshell}

The elements of the gauge group $\mathfrak{G}$ can also be seen as $G$-valued fields defined on $P$. Such an element $g$ is then transformed under the action of another element $h$ as $g^{h}=h^{-1} g h$. Let $K$ be a subgroup of $G$, possibly $G$ itself. A dressing field is a locally defined $G$-valued field $u$ on $P$, which transforms under a gauge transformation $k \in K$ as $u^{k}=k^{-1} u$. Thus, $u \notin \mathfrak{G}$.

The existence of such a field ensures that the following composite fields:

- $\omega^{u}:=u^{-1} \omega u+u^{-1} d u$,

- $\Omega^{u}:=u^{-1} \Omega u$,

- $\xi^{u}:=\rho(u)^{-1} u$,

are then $K$-gauge invariant as it can be checked by a straightforward computation. The fact that these fields are now $K$-invariant is interpreted saying that actually, the subgroup $K$ does not act anymore on the fields.

Thus, if one re-writes the theory (i.e. the gauge invariant action $S[\omega, \xi]$ ) in the new variables, one gets a theory for which the $K$-symmetry has been erased. It is a mere reconfiguration of the fields which redistributes the degrees of freedom of the theory. The latter are computed as follows: let \#TOT, \# $\Phi$, $\# \mathrm{G}$ and $\#(\Theta=0)$ be respectively the total number of degrees of freedom, the degrees of freedom related to the fields $(\omega$ and $\xi$ ) of the theory, the dimension of the symmetry group $G$, and the number of constraint equations. Then:

$$
\# \mathrm{TOT}=\# \Phi-\# \mathrm{G}-\#(\Theta=0) \text {. }
$$

For example, if the operation of dressing leaves invariant the constraint equations, in the new variables the theory will have less symmetry and then necessarily "less fields", i.e. less degrees of freedom coming from the fields.

Let us present now the two examples announced in the introduction. 


\section{The Higgs mechanism of the SM as a dressing field reduction.}

In the Standard Model, the electroweak sector is governed by the symmetry group $G=U(1) \times S U(2)$. The $S U(2)$-symmetry prevents the action from having mass terms for the weak bosons. Thus, one has to find a way to erase the $S U(2)$-symmetry. Let us present first the usual version, as developed by many authors in the 60's. Then, one presents another interpretation, developed in [1], based on the dressing field method.

\subsection{Usual Viewpoint}

The idea is to suppose the existence of a complex scalar field $\Phi: M \rightarrow \mathbb{C}^{2}$ embedded in the potential $V(\Phi)=-\mu^{2} \Phi^{\dagger} \Phi-\lambda\left(\Phi^{\dagger} \Phi\right)^{2}$, with $\lambda>0$, which spontaneously gets the value $\Phi_{\text {min }}$ which minimises the potential $V(\Phi)$. This value depends on the form of the potential, i.e. of the sign of $\mu^{2}$. For $\mu^{2}>0$, $\Phi_{\text {min }}=0$ and the choice $\Phi=0$ is unique, and viewed as a point in $\mathbb{C}^{2}$, it is still $S U(2)$-invariant. However, for $\mu^{2}<0, \Phi_{\text {min }} \neq 0$, and $\Phi$ has to "make a choice" (hence the term spontaneous) between a subset of correponding points in $\mathbb{C}^{2}$. It turns out that a particular point is no more $S U(2)$-invariant, and this phenomenon breaks the symmetry. The scalar field then reads $\Phi=$ $\Phi_{\text {min }}+H$, and the fluctuation $H$ is interpreted as a particle, the Higgs particle which has been discovered in the LHC in 2012. The constant part $\Phi_{\min }$ couples with other fields, giving them mass.

Thus, the generation of masses in the usual viewpoint is deeply related to the $S U(2)$-symmetry breaking. One shall see now that it is actually possible to reinterpret the Higgs mecanism without calling on a spontaneous symmetry breaking, but merely by viewing it as a dressing.

\section{2. $S U(2)$-erasing without symmetry breaking}

Let us take the same initial data as in the usual viewpoint. We are going to show that $S U(2)$ is actually always erasable by a dressing, u, built out of the scalar field $\Phi$.

The first step is to write the polar decomposition of $\Phi$ as an element of $\mathbb{C}^{2}$ : there exists $u \in S U(2)$ such that $\Phi=u \eta$, with $\eta=\left(\begin{array}{c}0 \\ \|\Phi\|\end{array}\right)$, with $\|\Phi\|^{2}:=\Phi^{\dagger} \Phi \in \mathbb{R}^{+}$. Due to the gauge transformation of $\Phi$ under $S U(2)$ (as a scalar doublet), the new variable $\eta$ is invariant under $S U(2)$, and $u$ transforms as: $u \rightarrow \beta^{-1} u$, with $\beta \in S U(2)$. Thus, $u$ is a dressing field. From this point, we already know that it is possible to erase the $S U(2)$-symmetry by dressing, whatever the value of $\mu^{2}$ is. $\eta=u^{-1} \Phi$ is the $S U(2)$-invariant composite field which takes the place of the original scalar field.

Now, one can generate masses for the weak fields by making $\eta$ fluctuating around its value which minimises $V(\eta)$. For $\mu^{2}>0, \eta_{\min }=0$ and mass terms are identically zero. For $\mu^{2}<0, \eta_{\min } \neq 0$, and moreover, there is no more actual "choice": $\eta$ being a positive real number, $V(\eta)$ is now a mere onevariable real valued function. Thus, the term "spontaneously" is no more 
relevant. Writing $\eta=\eta_{\min }+H$ leads to the same conclusions as in the usual viewpoint.

The only difference is in the physical interpretation: here, one has seen that the generation of masses is totally decorelated from the symmetry breaking. The latter is not seen as a physical phenomenon which historically occured. Rather, the original $S U(2)$-symmetry appears to be an artefact due to field variables in which the theory is originally written, and can be structurally erased by using new field variables. The dressing field method is a systematic way of finding such new field variables which simplify the theory.

Let us now present another example for which the dressing field method applies: the top-down construction of Twistor (and Tractor) bundles and connections.

\section{Twistors as composite fields from conformal Cartan geometry}

The whole construction being technical, one only sketches it. The interested reader is highly recommended to take a look at [2] (Tractors) and [3] (Twistors).

\subsection{The usual bottom-up construction}

Twistors are for conformal geometry what spinors are for Lorenztian geometry. These objects are usually obtained following a "bottom-up construction" over a conformal manifold $(M,[g])$, where $[g]=\left\{\lambda g, \lambda \in \mathcal{C}^{\infty}\left(M, \mathbb{R}^{+*}\right)\right\}$, for a Lorentzian metric $g$. As in Penrose's work, for example ([5], [6]), one takes a $\mathbb{C}^{2}$-valued field $\omega^{B}$ which satisfies the Twistor equation: $\nabla_{A A^{\prime}} \omega^{B}-$ $\frac{1}{2} \delta_{A}^{B} \nabla_{C A^{\prime}} \omega^{C}=0$, and then constructs a closed system by introducing another $\mathbb{C}^{2}$-valued field $\pi_{A^{\prime}}:=\frac{i}{2} \nabla_{C A^{\prime}} \omega^{C}$ :

- $\nabla_{A A^{\prime}} \omega^{B}+i \frac{1}{2} \delta_{A}^{B} \pi_{A^{\prime}}=0$,

- $\nabla_{A A^{\prime}} \pi_{B^{\prime}}-i \bar{P}_{A A^{\prime} B B^{\prime}} \omega^{B}=0$,

with $\bar{P}_{A A^{\prime} B B^{\prime}}$ corresponding to the Schouten tensor $P_{a b}=-\frac{1}{2}\left(R_{a b}-\frac{1}{6} R g_{a b}\right)$. One then encompasses the whole construction in the new definitions:

- $Z^{\alpha}:=\left(\omega^{B}, \pi_{A^{\prime}}\right) \in \mathbb{C}^{4}$,

- $\nabla_{A A^{\prime}}^{T} Z^{\alpha}=0$ with $\nabla_{A A^{\prime}}^{T}:=\nabla_{A A^{\prime}} \mathbb{I}_{4}+\left(\begin{array}{cc}0 & i \delta_{A}^{B} \\ -i \bar{P}_{A A^{\prime} B B^{\prime}} & 0\end{array}\right)$,

where $\nabla_{A A^{\prime}}^{T}$ is the Twistor connection and $Z^{\alpha}$ is a $\mathbb{C}^{4}$-valued Twistor. By construction, these equations are conformally invariant (i.e. well-defined on $(M,[g]))$. Yet, the objects like $\nabla_{A A^{\prime}}^{T}$ and $Z^{\alpha}$ are conformally covariant, and one can compute the corresponding transformation laws under a conformal rescaling of the metric. One can then consider a general Twistor $Z^{\alpha}$, i.e. an object such that $\nabla_{A A^{\prime}}^{T} Z^{\alpha}$ does not necessarily vanish, and which transforms with the same laws, which are:

$$
\hat{Z}^{\alpha}=\left(\begin{array}{cc}
\mathbb{I}_{2} & 0 \\
i \Upsilon_{A A^{\prime}} & \mathbb{I}_{2}
\end{array}\right) Z^{\alpha} \text { and } \widehat{\nabla_{A A^{\prime}}^{T} Z^{\alpha}}=\left(\begin{array}{cc}
\mathbb{I}_{2} & 0 \\
i \Upsilon_{A A^{\prime}} & \mathbb{I}_{2}
\end{array}\right) \nabla_{A A^{\prime}}^{T} Z^{\alpha}
$$


under $g_{\mu \nu} \rightarrow z^{2} g_{\mu \nu}$, with $\Upsilon_{A A^{\prime}}$ corresponding to $\Upsilon_{a}=\partial_{a} \ln (z)$.

Twistors are thus sections of a vector bundle with fibre $\mathbb{C}^{4}$, transforming under the action of a certain group represented by elements of the form $\left(\begin{array}{cc}\mathbb{I}_{2} & 0 \\ i \Upsilon_{A A^{\prime}} & \mathbb{I}_{2}\end{array}\right)$. One now presents another construction which is based on the dressing field method.

\subsection{A top-down construction via dressing field method}

A Cartan geometry over a manifold $M$ is a way of implementing local external symmetries (like Lorentz, projective, ...) in the form of usual internal ones. It is used to write gravitation theories (i.e. theories in which the geometry of the base manifold is dynamic) in the form of usual gauge theories. From a mathematical point of view, a Cartan geometry expresses a given geometric structure over $M$ into the form a principal bundle endowed with a so-called Cartan connection, slightly different from the usual notion of Ehresmann connection.

For example, the data of a Lorentzian metric on a manifold $M$ is equivalent to the data of a torsionfree Cartan connection over a $H$-principal bundle, $H$ being the Lorentz group. Following the same idea, a conformal manifold $(M,[g])$ can be seen as a normal conformal Cartan connection $\varpi$ over a $H$-principal bundle $P$, with $H$ defined as follows. Let $G:=S O(2,4) /( \pm \mathbb{I})$ be the conformal group, and $M_{0}:=S^{1} \times S^{3} / \mathbb{Z}_{2}$ the conformal compactification of Minkowski spacetime, which is homogeneous with respect to the action of $G$. The corresponding Lie group $H$ is then the stabilizer of a point of $M_{0}$. One takes then the complex $\mathbb{C}^{4}$-representation of these groups. A Twistor should be a section of the associated vector bundle $P \times{ }_{\bar{H}} \mathbb{C}^{4}$, where $\bar{H}$ is the complex representation of $H$. It turns out that as it stands, the structure does not reproduce the Twistor space and connection, for it does not imply the same transformation law.

To recover it, one has to apply the dressing field method, with dressing fields built out of the conformal Cartan connection. In doing so, one can erase some parts of the original structure group $H$, and end up with composite fields which transform under a modified transformation law corresponding to the residual action of $H$. Twistors as previously defined then appear from this construction, with a slight modification: the residual symmetry group does not act (on the composite fields) through a representation of the Weyl group $\mathbb{R}_{+}^{*}$, but via something more complicated called a $1-\alpha$-cocyle, see [3], sections 4.2.2.

The fact is that in our procedure, no arbitrary choice is made: one just takes the "rigid" normal conformal Cartan geometry and applies to it the dressing field method. Everything is "already there". One is just playing with objects which naturally belong to the geometry. On the contrary, in the usual construction, some ansatz are taken to simplify the transformation laws, rendering the construction more arbitrary, even if it remains, of course, totally coherent. 
Finally, let us remark that the same thing has been done also for Tractors, which appear to be merely the real version of Twistors ([2]).

\section{Conclusion}

In this presentation of the dressing field method, it has been shown that it can apply in a quite wide range of different cases. Once one works on a principal bundle equiped with a connection (of Erhesmann or Cartan type), one can try to investigate if it is possible to build a dressing field out of the fields to erase the action of the symmetry group or one of its subgroups. A first hint can be given by counting how are initially distributed the degrees of freedom, and how/if they could be distributed differently. Then, if the answer is positive, one can start to search for a field transforming on the right way to be a dressing field.

More than just giving a way of simplifying the writing of physical theories, it often offers a new insight into some already known constructions. In the case of the SM, it gives a natural and new interpretation of the generation of masses. In the case of Twistors (or even Tractors), it offers a new view of the geometric nature of these objects and of their underlying structure.

\section{Acknowledgments}

This paper stems from a joint work of the author and his colleague Jordan François and his supervisors Serge Lazzarini and Thierry Masson. Many thanks go to Jordan François and Serge Lazzarini for having read this paper and suggested useful corrections.

\section{References}

[1] T. Masson and J.-C. Wallet, A remark on the spontaneous symmetry breaking in the standard model arXiv:1001.1176 (2010).

[2] J.Attard and J. François, Tractors and Twistors from conformal Cartan geometry: a gauge theoretic approach. I. Tractors. arXiv:1609.07307, (2017).

[3] J.Attard and J. François, Tractors and Twistors from conformal Cartan geometry: a gauge theoretic approach. II. Twistors. Classical and Quantum Gravity, (2017).

[4] J.Attard, J. François, T.Masson and S.Lazzarini The dressing field method of gauge symmetry reduction: a review with examples Journal of Geometric Method in Modern Physics, (2017).

[5] R.Penrose Twistor algebra J. Math. Phys., 8:345 (1967).

[6] R.Penrose Twistor quantization and curved space-time Int. J. Theor. Phys., 1:6199 (1968).

[7] J. François, S.Lazzarini and T.Masson Residual Weyl symmetry out of conformal geometry and its BRS structure JHEP09 (2015) 195.

[8] J.Attard, J. François and S.Lazzarini Weyl Gravity and Cartan Geometry Physical Review D, (2015). 


\author{
Jeremy Attard \\ Centre de Physique Théorique \\ Campus de Luminy, Case 907 \\ 163 Avenue de Luminy \\ 13288 Marseille Cedex 9, France \\ e-mail: jeremy . attard@cpt . univ-mrs.fr
}

